\title{
Probing the symmetry energy from the nuclear isoscaling
}

\author{
S. R. Souza, ${ }^{1,2}$ M. B. Tsang, ${ }^{3}$ R. Donangelo, ${ }^{1}$ W. G. Lynch, ${ }^{3}$ and A. W. Steiner ${ }^{3}$ \\ ${ }^{1}$ Instituto de Física, Universidade Federal do Rio de Janeiro Cidade Universitária, CP 68528, 21941972, Rio de Janeiro, Brazil \\ ${ }^{2}$ Instituto de Física, Universidade Federal do Rio Grande do Sul Av. Bento Gonçalves 9500, CP 15051, 91501-970, Porto Alegre, Brazil \\ ${ }^{3}$ Joint Institute for Nuclear Astrophysics, National Superconducting Cyclotron Laboratory, and the Department of Physics and Astronomy, \\ Michigan State University, East Lansing, Michigan 48824, USA
}

(Received 8 April 2008; published 16 July 2008)

\begin{abstract}
Using different parametrizations of the nuclear mass formula, we study the sensitivity of the isoscaling parameters to the mass formula employed in grand-canonical calculations. Previous works on isoscaling have suggested that the symmetry energy implied in such calculations is anomalously smaller than that suggested by fits to nuclear masses. We show that surface corrections to the symmetry energy naturally broadens the isotopic distribution thus allowing for values of the symmetry energy which more closely match those obtained from nuclear masses.
\end{abstract}

PACS number(s): 25.70.Pq, 21.30.Fe, 21.65.Ef, 24.60.-k

\section{INTRODUCTION}

Understanding multifragmentation, the breakup of nuclei into many fragments, has challenged theorists and experimentalists for almost three decades [1-5]. This phenomenon can be observed in both relativistic light-ion collisions and in central nucleus-nucleus collisions, even though the role of reaction dynamics in these two domains can be quite different. The consequential variation in the mixture of statistical and timedependent phenomena in multifragmentation complicates the application of the traditional theoretical tools. Nonetheless, statistical techniques have been successfully applied to calculate the relative production of various fragments [3-7], even though the fundamental statistical parameters such as temperature or density are not external constraints, but must be inferred from data (see $[4,5,8,9]$, and references therein).

It is therefore natural to apply statistical methods to fragment isotope distributions and to their isoscaling behavior $[10,11]$. The latter term refers to the scaling properties observed in Refs. [10,11], that govern the experimentally observed ratios between the yields $Y_{i}(N, Z)$ associated with an emitted fragment of atomic and neutron numbers $Z$ and $N$, respectively, produced in two similar reactions, labeled $i=1,2$ :

$$
R_{21}(N, Z)=Y_{2}(N, Z) / Y_{1}(N, Z) \propto \exp (\alpha N+\beta Z),
$$

where $\alpha$, and $\beta$ are the isoscaling parameters. The isoscaling of fragment isotopic yields has also been observed in deep inelastic collisions, evaporation processes, as well as in nuclear multifragmentation reactions [11-13]. It has been exploited by many authors to obtain information on observables related to the multifragment emission [11,14-17]. In particular, a link between the symmetry energy coefficient $C_{\text {sym }}$ and the isoscaling parameter $\alpha$ has been suggested in Refs. [11,14],

$$
\alpha=\frac{4 C_{\mathrm{sym}}}{T}\left(\frac{Z_{1}^{2}}{A_{1}^{2}}-\frac{Z_{2}^{2}}{A_{2}^{2}}\right) .
$$

If a reaction allows straightforward application of statistical approaches, it may be possible to obtain empirical constraints on $C_{\text {sym }}$, at different values of the breakup density and temperature $T$, from the knowledge of the decaying sources $\left(A_{1}, Z_{1}\right)$ and $\left(A_{2}, Z_{2}\right)$ or, the proton fractions, $Z_{1} / A_{1}$ and $Z_{2} / A_{2}$, together with $\alpha$ and $T$. To explore this possibility, many studies along this line have been carried out, which rely directly [17] or indirectly [14,18-21] on the close connection between the symmetry energy and the isoscaling parameter $\alpha$. Such studies require explicit assumptions about the form for the symmetry free energy and about how the symmetry free energy depends on temperature.

In this work, we investigate these assumptions. We explore the sensitivity of $\alpha$ to the mass formula employed in statistical calculations. In addition to the standard parametrization of the binding energy [14], we also consider mass formulas in which the surface effects in the symmetry energy are included $[22,23]$. We examine the rigor of present extractions of the temperature dependence of the symmetry energy from isoscaling analyses and whether such analyses presently provide unambiguous information on this temperature dependence. In Sec. II, we present the statistical treatment employed in this work, as well as the mass formulas we use. The sensitivity of the isoscaling parameters to the binding energy is then investigated in Sec. III. Our main conclusions are delineated in Sec. IV.

\section{THE MODEL}

The statistical multifragmentation model (SMM) [24-26] is based on a scenario in which a hot source with mass and atomic numbers $A_{0}$ and $Z_{0}$, respectively, at temperature $T$, expands to a density $\rho=\rho_{0} /(1+\chi)$ and undergoes a prompt breakup. The normal nuclear density is denoted by $\rho_{0}$ and $1 / \chi$ corresponds to the ratio between the breakup volume and that available to the fragments. The properties of the system are calculated at the freeze-out stage, where it is explicitly assumed that, except for the Coulomb repulsion, there is no interaction among the fragments. Some interactions of the fragments with 
the surrounding light particle gas are incorporated via the temperature dependence of the nuclear surface energy. Within the SMM approach, the total Helmholtz free energy of the system is approximated by

$$
\begin{aligned}
F(T, V)= & n_{1}\left(f_{1}+f_{1}^{\text {trans }}\right)+\cdots+n_{M}\left(f_{M}+f_{M}^{\text {trans }}\right) \\
& +\frac{C_{\mathrm{c}}}{(1+\chi)^{1 / 3}} \frac{\left(\sum_{i} n_{i} Z_{i}\right)^{2}}{\left(\sum_{i} n_{i} A_{i}\right)^{1 / 3}}
\end{aligned}
$$

where $n_{i}$ denotes the multiplicity of species $i, f_{i}$ and $f_{i}^{\text {trans }}$ respectively stand for the internal and translational contributions of a fragment of species $i$ to the free energy. The last term in the above expression corresponds to the homogeneously charged sphere which, with the other terms contained in $f_{i}$, give the Wigner-Seitz approximation to the Coulomb interaction among the fragments [24,27]. The coefficient $C_{c}$ is taken from a mass formula (see below). The species multiplicities are constrained by the properties of the decaying source:

$$
\begin{aligned}
& A_{0}=\sum_{i}^{M} A_{i} n_{i}, \\
& Z_{0}=\sum_{i}^{M} Z_{i} n_{i} .
\end{aligned}
$$

\section{A. The grand canonical approach}

For clarity, we use the grand-canonical version of SMM, from which analytical expressions for the average fragment multiplicities can be easily obtained.

By construction, the terms involving $f_{i}(T, V)$ have no explicit or implicit dependence on $n_{i}$, since it has contributions only from the binding energy $B_{i}$, the internal excitation energy of the fragment and the remaining Wigner-Seitz Coulomb terms of Eq. (3). Thus, at this point, one only needs the explicit expression for $f_{i}^{\text {trans }}$ which, through the Stirling's formula, $\log n ! \approx n \log n-n$, can be written as

$f_{i}^{\text {trans }}\left(T, V, n_{i}\right)=-T\left[\log \left(\frac{g_{i} V_{f}}{\lambda_{T}^{3}} A_{i}^{3 / 2}\right)-\log n_{i}+1\right]$,

where $\lambda_{T}=\sqrt{2 \pi \hbar^{2} / m T}, m$ is the nucleon mass, $g_{i}$ denotes the spin degeneracy factor, and $V_{f}$ stands for the free volume.

The average fragment distribution may be obtained by minimizing the total free energy of the system with respect to $\left\{n_{k}\right\}$, subject to the constraints (4)-(5):

$$
\frac{\partial \mathcal{F}}{\partial n_{k}}=0
$$

where

$\mathcal{F}=F+\mu_{B}\left[A_{0}-\sum_{i} A_{i} n_{i}\right]+\mu_{Q}\left[Z_{0}-\sum_{i} Z_{i} n_{i}\right]$,

and $\mu_{Q}$ and $\mu_{B}$ are the Lagrange multipliers, which turn out to be the charge and baryon chemical potentials, respectively.
Thus, the above relations lead to

$$
\begin{aligned}
0= & f_{k}-T\left[\log \left(\frac{g_{i} V_{f}}{\lambda_{T}^{3}} A_{i}^{3 / 2}\right)-\log n_{i}+1\right] \\
& +T+\frac{2 Z_{k} C_{\mathrm{c}}}{(1+\chi)^{1 / 3}} \frac{\sum_{i} n_{i} Z_{i}}{\left(\sum_{i} n_{i} A_{i}\right)^{1 / 3}} \\
& -\frac{A_{k} C_{\mathrm{c}}}{3(1+\chi)^{1 / 3}} \frac{\left(\sum_{i} n_{i} Z_{i}\right)^{2}}{\left(\sum_{i} n_{i} A_{i}\right)^{4 / 3}}-\mu_{B} A_{k}-\mu_{Q} Z_{k} .
\end{aligned}
$$

Upon using Eqs. (4) and (5), one finally arrives at

$$
\begin{aligned}
n_{k}= & \frac{g_{k} V_{f} A_{k}^{3 / 2}}{\lambda_{T}^{3}} \exp \left\{-\left(f_{k}-\mu_{B} A_{k}-\mu_{Q} Z_{k}\right.\right. \\
& \left.\left.+\frac{2 Z_{k} Z_{0} C_{\mathrm{c}}}{A_{0}^{1 / 3}(1+\chi)^{1 / 3}}-\frac{A_{k} Z_{0}^{2} C_{\mathrm{c}}}{3 A_{0}^{4 / 3}(1+\chi)^{1 / 3}}\right) / T\right\} .
\end{aligned}
$$

It should be noticed that the last two terms in the above expression arise from the homogeneous term of the WignerSeitz approximation. Since the chemical potentials can be redefined to absorb these terms, they do not play a role in the determination of the particle multiplicity. However, they modify the relation between $\alpha$ and the mass formula, inasmuch as the difference between the chemical potentials in reactions 1 and 2 is the relevant quantity to the calculation $[11,14]$. Nevertheless, since the considered sources are usually similar, the symmetry energy still gives the main contribution to $\alpha$ and, therefore, this correction is neglected.

The internal free energy of the species $i$ is given by

$$
\begin{aligned}
f_{i}= & -B_{i}-\frac{T^{2}}{\epsilon_{0}} A_{i}+\beta_{0} A_{i}^{2 / 3}\left[\left(\frac{T_{c}^{2}-T^{2}}{T_{c}^{2}+T^{2}}\right)^{5 / 4}-1\right] \\
& -C_{\mathrm{c}} \frac{Z_{i}^{2}}{A_{i}^{1 / 3}} \frac{1}{(1+\chi)^{1 / 3}},
\end{aligned}
$$

where $B_{i}$ denotes the binding energy of the fragment, $\epsilon_{0}=$ $16 \mathrm{MeV}, \beta_{0}=18 \mathrm{MeV}$, and $T_{c}=18 \mathrm{MeV}$. The last term in the above relation is associated with the Wigner-Seitz correction, whereas the Coulomb self energy of the fragment is already included in $B_{i}$. The form of the suppression of the surface energy as $T \rightarrow T_{C}$ was determined in Ref. [24], by matching the microscopic results obtained in Ref. [28]. Very light fragments, $A<5$, are considered as point particles and therefore contribute to $f_{i}$ only through their Coulomb and binding energies, where the latter are taken from empirical data, as well as their spin degeneracy factors $g_{i}$. The exception is the alpha particle, in which case, one suppresses the surface contribution to the excited states. For all heavier nuclei, $A \geqslant 5, B_{i}$ is computed through a given mass formula and it is assumed that $g_{i}=1$ as the contribution to the statistical weight is, to some extent, taken into account by the excitation part of the free energy.

The chemical potentials $\mu_{Q}$ and $\mu_{B}$ are obtained by imposing the constraints given by Eqs. (4) and (5). In the numerical calculations, the relative error on the mass or proton numbers is required to be better than $10^{-10}$ in each case. 


\section{B. The binding energy}

The binding energy of nuclei plays an important role in the determination of the relative yields produced in the breakup of a nuclear system [23] and therefore it affects the isoscaling parameters. To investigate this point, we consider three different mass formulas based on the liquid drop model (LDM) [29]:

$$
B_{A, Z}=C_{v} A-C_{s} A^{2 / 3}-C_{c} \frac{Z^{2}}{A^{1 / 3}}+C_{d} \frac{Z^{2}}{A}+\delta_{A, Z} A^{-1 / 2},
$$

where

$$
\begin{aligned}
C_{\mathrm{c}} & =\frac{a_{\mathrm{c}}}{1+\Delta}, \\
C_{i} & =a_{i}\left[1-k_{i}\left(\frac{A-2 Z}{A}\right)^{2}\right],
\end{aligned}
$$

and $i=v, s$ corresponds to volume and surface terms, respectively. Notice that $C_{v}$ and $C_{s}$ might include contributions associated with the symmetry energy, as we discuss below. The following two terms correspond to the ordinary Coulomb reduction of the binding energy and its correction due to the surface diffuseness, respectively. In the first two models we consider below, $\Delta=0$, so the coefficient $C_{\mathrm{c}}=a_{\mathrm{c}}$ is constant. This is not the case for the last mass formula discussed below. The pairing coefficient has the usual meaning:

$$
\delta_{A, Z}=\left\{\begin{array}{cl}
+C_{p}, & N \text { and } Z \text { even } \\
0, & A \text { odd } \\
-C_{p}, & N \text { and } Z \text { odd }
\end{array},\right.
$$

where $N=A-Z$ denotes the neutron number.

The symmetry energy has contributions from the surface and bulk terms, which can be easily identified by grouping the corresponding factors from the formula:

$$
E_{\mathrm{sym}}=-C_{\mathrm{sym}} \frac{(A-2 Z)^{2}}{A},
$$

where

$$
C_{\mathrm{sym}} \equiv a_{v} k_{v}-\frac{a_{s} k_{s}}{A^{1 / 3}} .
$$

The simplest form of this mass formula, in which $k_{s}=0$, $C_{d}=0$, has been used in many statistical calculations $[6,14]$ and will be labeled henceforth as LDM1, whereas the complete formula, used in Refs. [23,30], is denoted as LDM2. Since the contribution associated with $C_{d}$ is small, the main difference between the two formulas is in the inclusion of surface effects in the symmetry energy in LDM2.

The third mass formula used in this work, LDM3, was introduced in Ref. [22], which also considers surface effects in the symmetry energy, but has a different functional form:

$$
\begin{aligned}
B_{A, Z}= & C_{v} A-C_{s} A^{2 / 3}-\frac{\alpha^{*}}{1+\frac{\alpha^{*}}{\beta^{*}} A^{-1 / 3}} \frac{(A-2 Z)^{2}}{A} \\
& -C_{c} \frac{Z^{2}}{A^{1 / 3}}+\delta_{A, Z} .
\end{aligned}
$$

This form reflects the well-known correlation between the bulk symmetry energy and the dependence of the surface energy on the isospin asymmetry [31-33]. Note that $\alpha^{*}$ and $\beta^{*}$ do not correspond to the isoscaling parameters. We chose these symbols so as to keep the notation as close as possible to that used in the original work. The Coulomb parameter $\Delta$, in this case, reads

$$
\Delta=\frac{1.90539}{A^{2 / 3}}-\frac{1}{1+\frac{\beta^{*}}{\alpha^{*}} A^{1 / 3}} \frac{A-2 Z}{6 Z} .
$$

Since this binding energy formula is more complex than that given by Eq. (12), the symmetry energy cannot be cast in the same form as Eq. (16). For large $A$ and small asymmetry, it can be approximated by Eq. (16), where

$$
C_{\mathrm{sym}} \approx \alpha^{*}-\frac{\alpha^{* 2} / \beta^{*}}{A^{1 / 3}}
$$

which is identical to the result found above.

As pointed out in Ref. [22], the inclusion of the normal surface and surface symmetry energies in the mass formula should be expected as it simply reflects the dependence of the nuclear energy functional or equation of state on the nuclear density. The magnitudes or functional forms of the surface symmetry energy, however, depend on the density dependence of the symmetry energy, which is poorly constrained. Investigations of the temperature dependence of the symmetry energy implicitly assume the temperature dependence to be a result of the underlying density dependence of the symmetry energy [11-13]. To model a temperature dependence without considering the surface symmetry energies of nuclei is intrinsically inconsistent.

The parameters of the three mass formulas have been fitted to the empirical binding energies given in Ref. [34], for $A>4$. In Fig. 1, we show the difference $\Delta B$ between the calculated $B_{A, Z}$ and the empirical values. In all the cases, one observes a fairly good overall agreement with the data, except in the regions where shell effects are relevant and cannot be taken into account by these simple formulas. It is important to emphasize that $\Delta B$ denotes the total difference and that it is not divided

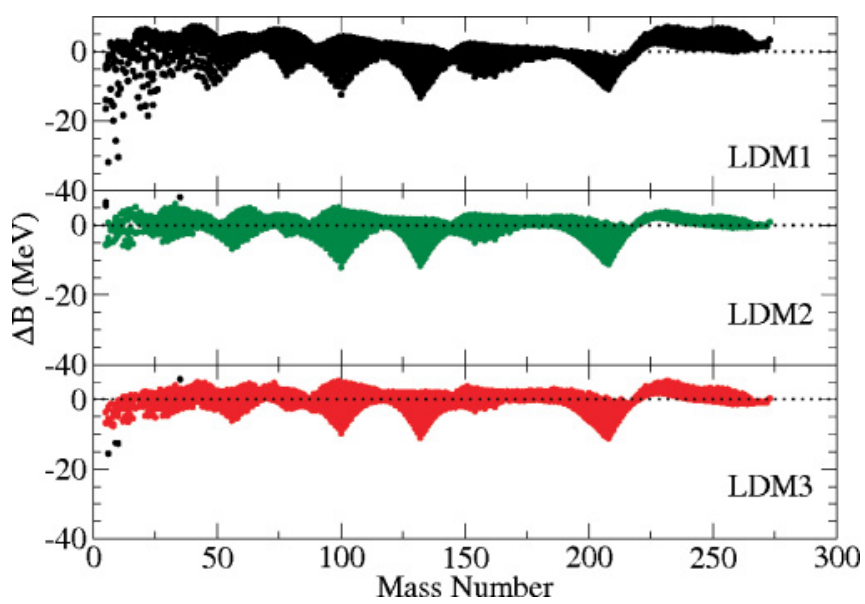

FIG. 1. (Color online) Difference between the calculated binding energies and the empirical values of Ref. [34] as a function of the mass number for the three mass formulas used in this work. 
TABLE I. Parameters of the liquid drop mass formulae used in this work. All the values are given in MeV.

\begin{tabular}{lccccccccc}
\hline \hline Label & $a_{v}$ & $a_{s}$ & $a_{c}$ & $C_{d}$ & $C_{p}$ & $a_{v} k_{v}$ & $a_{s} k_{s}$ & $\alpha^{*}$ & $\beta^{*}$ \\
\hline LDM1 & 15.2692 & 16.038 & 0.68698 & 0.0 & 11.277 & 22.3918 & 0.0 & na & na \\
LDM2 & 15.6658 & 18.9952 & 0.72053 & 1.74859 & 10.857 & 27.7976 & 33.7053 & na & na \\
LDM3 & 15.5586 & 18.2369 & 0.70521 & na & 11.012 & na & na & 27.1508 & 16.6412 \\
\hline \hline
\end{tabular}

by the mass number, as this quantity is usually displayed. Larger discrepancies are observed for light nuclei, $A<25$, with the LDM1 than for LDM2 or LDM3, because surface effects in the symmetry energy, which are more important in the light fragments, is disregarded in the LDM1 mass formula. The other mass formulas, LDM2 and LDM3, are both more accurate than LDM1, and LDM2 is slightly more accurate than LDM3.

The best fit parameters are listed in Table I. Although pairing is switched off in the calculations presented in the next section, we include it here for completeness. One may notice that the common parameters in LDM2 and LDM3 are very similar. The best fit value of $\alpha^{*}$ is very close to $a_{v} k_{v}$, which explains the resemblance between the predictions of the two mass formulas. Deviations should be expected for light masses, where the factor proportional to $1 / A^{1 / 3}$ in Eqs. (17) and (20) becomes non-negligible, because $\alpha^{* 2} / \beta^{*} \approx 44.3 \mathrm{MeV}$ differs appreciably from $a_{s} k_{s} \approx 33.7 \mathrm{MeV}$.

\section{RESULTS AND DISCUSSION}

The grand canonical model presented above is now applied to the description of the breakup of the ${ }^{112} \mathrm{Sn}$ and the ${ }^{124} \mathrm{Sn}$ nuclei at $\rho / \rho_{0}=1 / 3$. In Fig. 2, we show the isoscaling parameter $\alpha$, obtained using the different mass formulas, as a function of the temperature. At $T=6 \mathrm{MeV}$, up to $30 \%$ reduction of the isoscaling parameter $\alpha$ can be obtained by including the surface effects in the symmetry energy (LDM2/LDM3) (see Fig. 2), while preserving the good

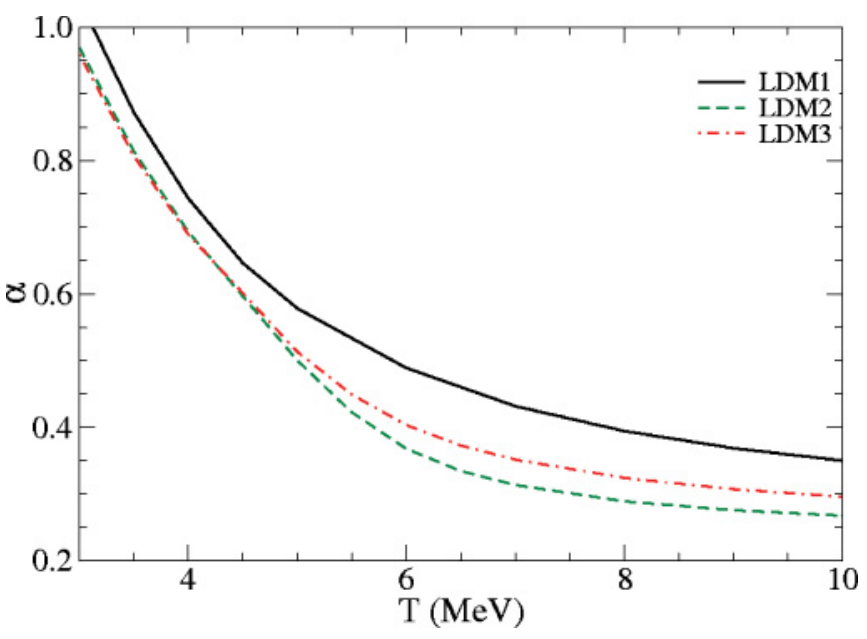

FIG. 2. (Color online) Isoscaling parameter $\alpha$ obtained using different binding energies in the grand-canonical calculations as a function of the temperature. agreement with the empirical binding energies. The final values for $\alpha$ are comparable to the data of Ref. [14], which suggest that one may be able to reproduce data by adopting a more accurate mass formula without grossly changing the symmetry energy values obtained from the fitting of the nuclear binding energies. Even so, the results reveal that distinct parametrizations of the surface symmetry energy energy do lead to appreciably different values of $\alpha$, in spite of the fact that the mass formulas are, to a large extent, equally accurate. So the parametrizations adopted here may still not be the optimal ones.

This differences in the predictions for $\alpha$ for the different mass models may be understood from the relationship between $\alpha$ and the symmetry energy in Eq. (2), as discussed in Refs. [11, 14]. At low temperatures, the chemical potentials are strongly influenced by the heavy fragments, which dominate the mass distribution. Since, in this mass region, the coefficient $C_{\text {sym }}$ in LDM2 and in LDM3 is nearly the same, as just discussed, Eq. (2) predicts that the isoscaling parameter $\alpha$ obtained with these two mass formulas should be very similar. The opposite holds in the case of the LDM1. One should note that Eq. (2) has been derived at very low temperatures [14]. As the temperature increases, more light fragments with $A<50$ are produced and the changes in the chemical potentials in the ${ }^{112} \mathrm{Sn}$ and ${ }^{124} \mathrm{Sn}$ systems are appreciably affected by them. Therefore, the binding energy differences observed in Fig. 1, in this lighter mass region, become very important. This explains the increase of the discrepancy between the values of $\alpha$ obtained using LDM2 and LDM3 at high temperatures. Eventually, our three curves will converge again at much higher temperatures $(T \gg 10 \mathrm{MeV})$, where only very light particles are produced. This latter convergence would occur because we use empirical binding energies in this mass region in the statistical calculations in all the cases.

The difference between the values of $\alpha$ obtained using LDM1 and LDM2/LDM3 is important for interpreting experimental data because in recent studies the symmetry energy coefficient has been adjusted in order to reproduce the experimental values of $\alpha$ [17]. Our results indicate that a large temperature dependence to the symmetry energy may not be necessary if a suitable parametrization of the binding energies are incorporated in the models. Nevertheless, we continue below to discuss other issues that may be important to consider if it still appears interesting to include an additional temperature dependence to the symmetry energy.

We now examine the consequences of changing $C_{\text {sym }}$ in statistical calculations, as has been made in different studies that focused on the isoscaling analysis [17-19,35] and on the isotopic distribution [36,37]. The top panel of Fig. 3 displays the parameter $\alpha$ obtained using LDM1 for different values 


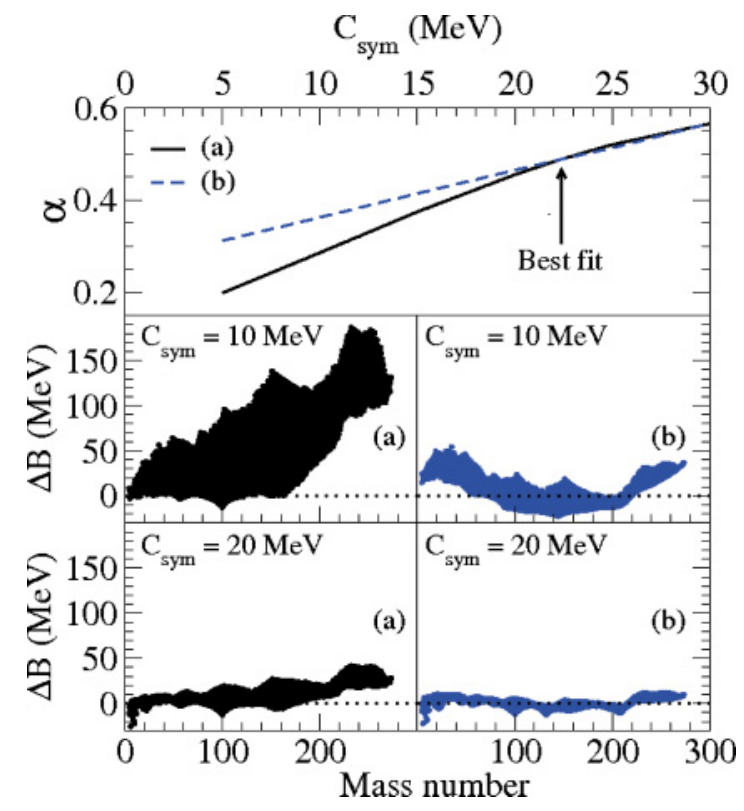

FIG. 3. (Color online) Upper panel: isoscaling parameter $\alpha$ obtained using LDM1 as a function of $C_{\mathrm{sym}}$. In case (a) all the LDM1 parameters are kept fixed, except for $C_{\text {sym }}$, whereas, in case (b), for each value of $C_{\text {sym }}$, the other LDM1 parameters are refitted to the empirical binding energies. Lower panels: $\Delta B$ for different values of $C_{\text {sym. }}$. The left panels correspond to case (a) and the right ones to case (b).

of $C_{\text {sym }}=a_{v} k_{v}$, for $T=6 \mathrm{MeV}$. The full line represents the results obtained in the approach of [17-19,35] and [36,37], which are labeled as case (a), where all the parameters of the mass formula are kept fixed, except for $C_{\text {sym }}$. The values for $\alpha$ decrease as $C_{\text {sym }}$ decreases, as noted by Refs. [17-19,35]. The left side of the lower two panels in Fig. 3 display the difference $\Delta B$ between the calculated $B_{A, Z}$, in case (a), and the empirical values. Reducing the symmetry energy coefficient to $C_{\text {sym }}=10 \mathrm{MeV}$, similar to the values deduced in Ref. [17], increases the binding of the most neutron-rich fragments of mass number 100 by up to $100 \mathrm{MeV}$ without changing the binding of the symmetric fragments. If the authors of Refs. [17-19,35] are correct, why does it occur? Without changing the nuclear density, such a large effect can only be due to changes in the interaction component of the symmetry energy; recent calculations of the temperature dependence for interaction component of the symmetry energy predicts a reduction in $C_{\text {sym }}$ of less than $5 \mathrm{MeV}$ [38-40].

Indeed, the authors of $[18,19,35]$, explain their reduction in $C_{\text {sym }}$ to be the result of a reduction of the internal densities of nuclei in their equilibrium calculations. Using this idea, these authors, in Ref. [18], tried to use the reduced values they extract for $C_{\text {sym }}$ to obtain experimental values for the symmetry energy of uniform matter at subsaturation density. However, in their SMM calculations, the matter in the fragmenting system is not uniform, and the reduction of the overall density of the multifragmenting systems is mainly due to the separation between the fragments. The fragments in their calculations are assumed to be at normal density and have the bulk, surface, and Coulomb energies of normal nuclei.
In order to accurately calculate the influence of reducing the density on the value of the symmetry energy coefficient $C_{\text {sym }}$, however, it is also necessary to consider the influence of that density change on the bulk, surface and Coulomb energies of the expanded nuclei. If the equilibrium central density of these expanded nuclei is not to be considered a free parameter, one would need to constrain it by the requirement of internal mechanical equilibrium within the fragmenting system, and then embed the resulting phenomenology within a modified SMM code. To compare to data, the influence of nuclear expansion on the sequential decay properties of the expanded nuclei would need to be considered. Otherwise the properties of the hot nuclei would be inconsistent with the properties of nuclei in the secondary decay stage. Taking all of this into account, the accurate modeling of expanded nuclei would represent a very significant modification of current SMM models. Consequently, it is difficult to accurately predict the results of such a project without doing it. For this reason, we decided not to speculate on this subject in the present paper.

Instead we ask whether significantly different results would be obtained with a model for the nuclear free energies that has a reduced value for $C_{\text {sym }}$, but does not exhibit the large overbinding of the heavy neutron-rich nuclei shown in the middle left panel of Fig. 3. We note that it is possible to reduce the overbinding for the heaviest neutron-rich isotopes by refitting the binding energy expression LDM1 used in the free energy calculation of hot nuclei to the measured masses. We have done this and show the results as case (b) in the right panels of Fig. 3. The dashed line in the upper panel of this figure shows the dependence of $\alpha$ on $C_{\text {sym }}$ to be rather similar to that for case (a). After refitting, the neutron-rich nuclei with $A<30$ are overbound by values ranging up to about $2 \mathrm{MeV} /$ nucleon, indicating a considerable enhancement in the attractive potential energy contribution for such neutron-rich nuclei.

The results for both case (a) and case (b) clearly show that $\alpha$ is strongly sensitive to $C_{\text {sym }}$, as reported previously [17]. For $C_{\text {sym }}>22 \mathrm{MeV}$, there is almost no difference whether one changes the other parameters of the mass formula, since the symmetry energy becomes so important that its contribution to the determination of $\alpha$ overwhelms the other terms. On the other hand, for smaller $C_{\text {sym }}$ values, the other terms of the mass formula, neglected in the derivation of Eq. (2), become non-negligible and therefore the two procedures lead to distinct results. Indeed, in the discussion presented in Ref. [14], it is clear that all terms in the mass formula enter in the expression. For the standard mass formula parametrizations, $C_{\text {sym }}$ gives the most important contribution to this expression. However, as one refits LDM1, for fixed $C_{\text {sym }}$, the values of the other terms change appreciably, in order to reduce the discrepancies with the observed binding energies. Thus, their contribution to Eq. (2) becomes non-negligible, when $C_{\text {sym }}$ is small, leading to the differences observed in the upper panel of Fig. 3.

For $C_{\text {sym }}$ below the best fit value, values for $\Delta B$ of the order of $2 \mathrm{MeV} /$ nucleon are obtained for light nuclei $A<20$ at $C_{\text {sym }}=10 \mathrm{MeV}$. We note that such light nuclei are the dominant species at high temperatures in any SMM calculation, whether for case (a), (b) or for the calculations with LDM1, LDM2, or LDM3 shown earlier. Thus, all attempts 


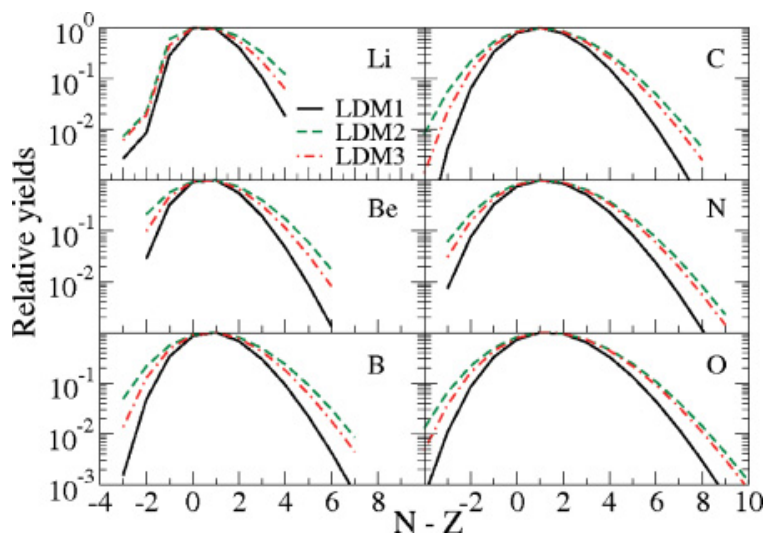

FIG. 4. (Color online) Isotopic distribution of fragments produced in the decay of the ${ }^{112} \mathrm{Sn}$ nucleus at $T=6 \mathrm{MeV}$.

within the SMM approach to explain the values of $\alpha$ reported by [17-19,35] involve modifying the free energies of the light fragments with $A<30$. The most important theoretically justified modification to these light fragment free energies is to include surface symmetry energy terms, to reflect the strong influence of the nuclear surface on the symmetry energies of light nuclei. The option of reducing $C_{\text {sym }}$ to low values like $C_{\text {sym }}=10 \mathrm{MeV}$, while leading to similar reductions in $\alpha$, does not seem to have a clear theoretical justification. More theoretical work is needed to explain why it is reasonable to expect such a large reduction in $C_{\text {sym }}$ and to justify the particular form of the reduction chosen in Refs. [17-19,35].

We note that $C_{\text {sym }}$ has also been reduced in different studies $[36,37]$ in order to obtain broader isotopic distributions. The inclusion of surface symmetry energy terms also has a similar effect in this case. We show, in Fig. 4, the isotopic distribution for some light nuclei, calculated for the breakup of the ${ }^{112} \mathrm{Sn}$ at $T=6 \mathrm{MeV}$, using the three different mass formulas discussed in this work, with the best fit parameters listed in table I. These results clearly show that the $A$ dependence of $C_{\text {sym }}$ in LDM2 and LDM3 leads to wider distributions as $C_{\text {sym }}$ becomes smaller for light nuclei, due to the influence of the symmetry energy.

\section{CONCLUDING REMARKS}

We have analyzed the role played by the mass formula used in statistical calculations to determine the isoscaling parameter $\alpha$, as well as in the isotopic distribution of fragments produced in the breakup of a thermally excited nucleus. Our results show that the reduction of the symmetry energy values [17-19,35-37], which best fit the empirical masses, may not be necessary to reproduce the experimental $\alpha$ and the isotopic distributions. Rather, the observed experimental reduction of the isoscaling parameter [17-19] can be reproduced if the surface corrections to the symmetry energy are included in the mass parametrization used in the statistical multifragmentation models. Since non-negligible deviations from the optimal value of $C_{\text {sym }}$ lead to large discrepancies between the theoretical and the empirical binding energies and free energies used in the model calculations, such changes should not be taken lightly especially if similar effects can be reproduced by the inclusion of surface effects in the mass formula. Certainly, some additional theoretical work is needed to justify the forms of the modifications to the symmetry energies that have been chosen in Refs. [17-19]. Furthermore, as pointed out in Ref. [41], improved treatments to the decay of the primary fragments than the usual Weisskopf approach $[42,43]$ also contribute to isotopic distributions with larger tails. Finally, we believe that our conclusions will not be affected by more realistic treatments which may introduce a temperature dependence to the symmetry energy since the corresponding effects, at a fixed density, are expected to be small [38-40].

\section{ACKNOWLEDGMENTS}

We would like to acknowledge CNPq, FAPERJ, and the PRONEX program under contract No. E-26/171.528/2006, for partial financial support. This work was supported in part by the National Science Foundation under Grant Nos. PHY-0606007 and INT-0228058. A.W.S. is supported by Joint Institute for Nuclear Astrophysics at MSU under NSF-PFC grant PHY 02-16783.
[1] B. Jakobsson, Z. Phys. A 307, 293 (1982).

[2] J. E. Finn, S. Agarwal, A. Bujak, J. Chuang, L. J. Gutay, A. S. Hirsch, R. W. Minich, N. T. Porile, R. P. Scharenberg, B. C. Stringfellow et al., Phys. Rev. Lett. 49, 1321 (1982).

[3] L. G. Moretto and G. J. Wozniak, Annu. Rev. Nucl. Part. Sci. 43, 379 (1993).

[4] S. Das Gupta, A. Z. Mekjian, and M. B. Tsang, Adv. Nucl. Phys. 26, 89 (2001).

[5] C. B. Das, S. Das Gupta, W. G. Lynch, A. Z. Mekjian, and M. B. Tsang, Phys. Rep. 406, 1 (2005).

[6] J. P. Bondorf, A. S. Botvina, A. S. Iljinov, I. N. Mihustin, and K. Sneppen, Phys. Rep. 257, 133 (1995).

[7] S. R. Souza, R. Donangelo, W. G. Lynch, and M. B. Tsang, Phys. Rev. C 76, 024614 (2007).

[8] A. Le Fèvre, O. Schapiro, and A. Chbihi, Nucl. Phys. A657, 446 (1999).
[9] J. B. Natowitz, K. Hagel, Y. Ma, M. Murray, L. Qin, S. Shlomo, R. Wada, and J. Wang, Phys. Rev. C 66, 031601(R) (2002).

[10] H. S. Xu, M. B. Tsang, T. X. Liu, X. D. Liu, W. G. Lynch, W. P. Tan, A. Vander Molen, G. V. A. Wagner, H. F. Xi, C. K. Gelbke et al., Phys. Rev. Lett. 85, 716 (2000).

[11] M. B. Tsang, W. A. Friedman, C. K. Gelbke, W. G. Lynch, G. Verde, and H. S. Xu, Phys. Rev. Lett. 86, 5023 (2001); M. B. Tsang, C. K. Gelbke, X. D. Liu, W. G. Lynch, W. P. Tan, G. Verde, H. S. Xu, W. A. Friedman, R. Donangelo, S. R. Souza, C. B. Das, S. Das Gupta, and D. Zhabinsky, Phys. Rev. C 64, 054615 (2001).

[12] D. V. Shetty, S. J. Yennello, E. Martin, A. Keksis, and G. A. Souliotis, Phys. Rev. C 68, 021602(R) (2003).

[13] G. A. Souliotis, D. V. Shetty, M. Veselsky, G. Chubarian, L. Trache, A. Keksis, E. Martin, and S. J. Yennello, Phys. Rev. C 68, 024605 (2003). 
[14] A. S. Botvina, O. V. Lozhkin, and W. Trautmann, Phys. Rev. C 65, 044610 (2002).

[15] Ad. R. Raduta and F. Gulminelli, Phys. Rev. C 75, 024605 (2007); Ad. R. Raduta and F. Gulminelli, Phys. Rev. C 75, 044605 (2007).

[16] S. R. Souza, R. Donangelo, W. G. Lynch, W. P. Tan, and M. B. Tsang, Phys. Rev. C 69, 031607(R) (2004).

[17] A. Le Fèvre, G. Auger, M. L. Begemann-Blaich, N. Bellaize, R. Bittiger, F. Bocage, B. Borderie, R. Bougault, B. Bouriquet, J. L. Charvet et al., Phys. Rev. Lett. 94, 162701 (2005).

[18] J. Iglio, D. V. Shetty, S. J. Yennello, G. A. Souliotis, M. Jandel, A. L. Keksis, S. N. Soisson, B. C. Stein, S. Wuenschel, and A. S. Botvina, Phys. Rev. C 74, 024605 (2006).

[19] D. V. Shetty, S. J. Yennello, and G. A. Souliotis, Phys. Rev. C 76, 024606 (2007).

[20] S. Kowalski, J. B. Natowitz, S. Shlomo, R. Wada, K. Hagel, J. Wang, T. Materna, Z. Chen, Y. G. Ma, L. Qin et al., Nucl. Phys. A787, 619c (2007).

[21] S. Kowalski, J. B. Natowitz, S. Shlomo, R. Wada, K. Hagel, J. Wang, T. Materna, Z. Chen, Y. G. M. L. Qin, A. S. Botvina et al., Phys. Rev. C 75, 014601 (2007).

[22] P. Danielewicz, Nucl. Phys. A727, 233 (2003).

[23] S. R. Souza, P. Danielewicz, S. Das Gupta, R. Donangelo, W. A. Friedman, W. G. Lynch, W. P. Tan, and M. B. Tsang, Phys. Rev. C 67, 051602(R) (2003).

[24] J. P. Bondorf, R. Donangelo, I. N. Mishustin, C. Pethick, H. Schutz, and K. Sneppen, Nucl. Phys. A443, 321 (1985).

[25] J. P. Bondorf, R. Donangelo, I. N. Mishustin, and H. Schutz, Nucl. Phys. A444, 460 (1985).

[26] K. Sneppen, Nucl. Phys. A470, 213 (1987).

[27] E. Wigner and F. Seitz, Phys. Rev. 46, 509 (1934).
[28] M. Brack, C. Guet, and H. B. Høakansson, Phys. Rep. 123, 275 (1985).

[29] M. A. Preston and R. K. Bhaduri, Structure of the Nucleus (Addison-Wesley, Reading, MA, 1975).

[30] W. P. Tan, S. R. Souza, R. J. Charity, R. Donangelo, W. G. Lynch, and M. B. Tsang, Phys. Rev. C 68, 034609 (2003).

[31] W. D. Myers and W. J. Swiatecki, Ann. Phys. (NY) 55, 395 (1969).

[32] J. M. Lattimer, C. J. Pethick, D. G. Ravenhall, and D. Q. Lamb, Nucl. Phys. A432, 646 (1985).

[33] J. M. Lattimer and F. D. Swesty, Nucl. Phys. A535, 331 (1991).

[34] G. Audi and A. H. Wapstra, Nucl. Phys. A595, 409 (1995).

[35] D. V. Shetty, A. S. Botvina, S. J. Yennello, G. A. Souliotis, E. Bell, and A. Keksis, Phys. Rev. C 71, 024602 (2005).

[36] N. Buyukcizmeci, R. Ogul, and A. S. Botvina, Eur. Phys. J. A 25, 57 (2005).

[37] A. S. Botvina, N. Buyukcizmeci, M. Erdogan, J. Łukasik, I. N. Mishustin, R. Ogul, and W. Trautmann, Phys. Rev. C 74, 044609 (2006).

[38] F. L. Braghin, Phys. Rev. C 71, 064303 (2005).

[39] F. L. Braghin, Nucl. Phys. A665, 13 (2000).

[40] J. Xu, Lie-Wen Chen, Bao-An Li, and Hong-Ru Ma, Phys. Rev. C 77, 014302 (2008).

[41] S. R. Souza, W. P. Tan, R. Donangelo, C. K. Gelbke, W. G. Lynch, and M. B. Tsang, Phys. Rev. C 62, 064607 (2000).

[42] A. S. Iljinov, I. N. Mishustin, J. P. Bondorf, R. Donangelo, and K. Sneppen, Nucl. Phys. A475, 663 (1987).

[43] S. R. Souza, R. Donangelo, W. G. Lynch, and M. B. Tsang, Phys. Rev. C 76, 024614 (2007). 